\title{
Le douzième colloque du groupe de recherche en communication des organisations (grec/o)
}

\section{Hugues Hotier}

\section{(2) OpenEdition}

1 Journals

Édition électronique

URL : http://journals.openedition.org/communicationorganisation/2792

DOI : 10.4000/communicationorganisation.2792

ISSN : $1775-3546$

Éditeur

Presses universitaires de Bordeaux

\section{Édition imprimée}

Date de publication : 1 novembre 2002

ISSN : 1168-5549

\section{Référence électronique}

Hugues Hotier, «Le douzième colloque du groupe de recherche en communication des organisations (grec/o) », Communication et organisation [En ligne], 22 | 2002, mis en ligne le 27 mars 2012, consulté le 01 mai 2019. URL : http://journals.openedition.org/communicationorganisation/2792 ; DOI : 10.4000/ communicationorganisation.2792

Ce document a été généré automatiquement le 1 mai 2019.

(c) Presses universitaires de Bordeaux 


\title{
Le douzième colloque du groupe de recherche en communication des organisations (grec/o)
}

\author{
Hugues Hotier
}

1 Entre la remise de la copie et la publication de l'article, il s'écoule plusieurs semaines et bien des choses peuvent se produire durant cette période. Au moment où j'écris ces lignes, fin avril, je ne suis pas sûr que des modifications n'interviendront pas dans le contenu de notre douzième colloque. Mais il parait certain que l'esprit que nous avons voulu donner à cette réunion internationale ne sera pas altéré. Encore que... Toujours au moment de l'écriture, une pneumopathie qui s'offre le luxe d'être atypique en plus de sa propension à la contagion nous vient de Chine alors que notre manifestation comporte une table ronde sur le développement des sciences de l'information et de la communication dans ce pays avec la participation de plusieurs universitaires chinois qui feront spécialement le déplacement de Bordeaux. Sans céder à la panique, force est de constater que l'épidémie est loin d'être maitrisée; au point que certaines compagnies aériennes. Egypt Air par exemple, annulent leurs vols vers la Chine. Il peut donc exister une incertitude. Mais, malgré les aléas inhérents à toute organisation, nous sommes des optimistes et le programme que vous trouverez ci-après doit être considéré comme définitif, les intervenants qui y figurent nous ayant confirmé leur participation.

2 Fondé en décembre 1986. le Groupe de Recherche en Communication des Organisations est une des trois composantes du «Centre d'Études des Médias » de l'Université Michel de Montaigne - Bordeaux 3 et. à ce titre, est reconnu en tant qu'« équipe d'accueil». Comprenez qu'il accueille des doctorants préparant des thèses en sciences de l'information et de la communication. Ils sont actuellement une quinzaine. Depuis 1995. le GREC/O fonctionne au rythme de programmes triennaux. Deux années et demie de travail mènent à la publication d'un ouvrage collectif qui joue le rôle de textes préparatoires à un colloque, lui-même suivi de la publication des actes sous la forme d'un numéro thématique de la revue Communication \& Organisation. Compte tenu du fonctionnement des universités selon des plans et des contrats quadriennaux imposés par 
le ministère, on pourrait trouver anormal de programmer la recherche à un rythme différent. En fait, les universitaires qui lisent cet article savent très bien que le ministère n'est pas un modèle de régularité ! Le dernier quadriennal de notre université a duré trois ans... Et l'on a même vu. il y a une dizaine d'années, sous prétexte d'un changement de majorité politique, un ministre nouvellement nommé déclarer qu'il n'était pas lié par les engagements contractuels pris par son prédécesseur. Notre rythme triennal nous satisfait. Gardons le sans nous préoccuper outre mesure de l'environnement politicoadministratif qui n'est pas à l'abri des errances et qui. on le constate particulièrement en ce moment, n'est pas aussi respectueux qu'on le souhaiterait de la recherche et des chercheurs.

On observe que, depuis 1995, nos travaux s'articulent autour d'un paradigme que nous avons défini par pur intérêt heuristique mais qui a eu pour effet de renforcer la cohérence de notre groupe. Ce que nous avons appelé induction s'est révélé être une thématique particulièrement féconde pour le colloque de 1997. Et quand nous avons choisi le non verbal comme thème pour la période 1997-2000. nous nous sommes rapidement aperçus que nous n'avions pas quitté l'induction; le colloque de mai 2000 intitulé "Non verbal, communication, organisation» et les actes qui ont été publiés l'attestent. Rechercher les modes de coexistence dans les organisations à travers le «traitement» réservé aux identités, à travers les traces ou à travers des modes de fonctionnement collectifs tels le projet, relève certainement aussi de l'induction dans la communication. Nous attendrons les débats du colloque pour l'affirmer.

Les textes préparatoires, fruits de la réflexion de plusieurs chercheurs du GREC/O, ont été rassemblés par Gino Gramaccia, professeur à l'Université Bordeaux I (sciences et technologies), et Elizabeth Gardère, ATER dans cette même université, qui a soutenu une thèse en communication organisationnelle particulièrement brillante à l'Université de Rennes en décembre 2002. On peut se les procurer en librairie (Coexister dans les mondes organisationnels, L'Harmattan, coll. Communication des organisations. 2003). reste à présenter le programme tel qu'il se présente le 22 avril, date de la rédaction de ce texte. 
Gino Gramaccia, chercheur au GREC/O. professeur de sciences de l'information et de la communication à l'Université Bordeaux 1 (sciences et techniques)

\subsection{Pause}

11.00 Axe 1 : le registre des identités, qui s'étend du cosmopolitisme assumé à l'acculturation forcée

Conférence introductive par Patrick Baudry, professeur de sociologie à l'Université Bordeaux 3

Deux ateliers simultanés dans lesquels seront réparties les communications suivantes :

Les réseaux inter organisationnels comme espace Je création Je connaissance

Alsones Balestrin. Université Fédérale du Rio Grande do Sul (Brésil) et Pierre Fayart, Université de Poitiers

Le rôle des organisations de la culture dans l'acculturation des pays en développement

Sandrine Basilico, IUT de Vichy et LAMIC, Université de Nice

Les cadres et les dirigeants: des interfaces fonctionnelles et symboliques dans les mondes organisationnels

Rosette et Jacques Bonnet, ENESAD (Dijon) et LIMSIC, Université de Bourgogne

Coexister dans la communauté européenne : organisations religieuses et prégnance symbolique

Stéfan Bratosin, Université Toulouse 3

L'identité salariale et l'acculturation à l'entreprise globalisée Bernard Floris. Université Grenoble 3

$$
\text { "Savoir être ", communication et enjeux identitaires Thomas Heller, Université Lille I }
$$

La mémoire organisationnelle en rupture avec la mémoire individuelle

André A. Lafrance. Chaire de relations publiques. Université du Québec à Montréal (Canada)

De l'impossible co-existence?

Philippe Saielli. Université de Valenciennes,

Isabelle Gillet, Université Lille 2

Le rôle des professionnels de la communication dans la gestion de la culture l'organisation

Laura Salamanca. Université Catholique de Louvain

La théorie de la justification: un modèle pour l'étude du travail en réseau dans le domaine psychomédico-social?

40 Isabelle Deliége, Groupe de Recherche en Médiation des Savoirs, Département de communication. Université Catholique de Louvain (Belgique)

$41 \quad 16.00$ - 18.00 Table ronde : les sciences de l'information et de la communication en Chine

Avec la participations de cinq professeurs de l'Université de Wuhan exerçant des responsabilités à l'Institut du Journalisme et de la Communication (IJC) : M. Luo Yicheng, directeur. MM. Shan Bo et Shy Yibin, directeurs adjoints. M. Zhang Jinhai, doyen du département de publicité et communication. M. Qiang Yuéxin, doyen du département de journalisme. 
Thèmes des communications dont les textes seront envoyés aux participants deux semaines avant le colloque :

- La communication interculturelle, les obstacles à son développement

- État actuel et perspectives du marché des médias en Chine dans le contexte interculturel de la mondialisation

- L'industrialisation des médias en Chine dans le contexte interculturel de la mondialisation

- Les formations supérieures au journalisme et à la communication en Chine

- Les nouvelles tendances de la recherche en sciences de l'information et de la communication en Chine

Animateur-modérateur: Patrice Legendre, $L$ 'Expression d'entreprise Interprètetraductrice : Zhijié Wang, Université de Wuhan et IUT d'Evry

Vendredi 13 juin

09.00 Axe 2 : le registre des inscriptions matérielles et spatiales, qui va d'un mode intensif à un mode extensif (condensation vs dilution, dissémination/agrégat vs conglomérat)

2 Conférence introductive par Paul Rasse, professeur de sciences de l'information et de la communication à l'Université de Nice.

Deux ateliers simultanés dans lesquels seront réparties les communications suivantes :

La communication au service Je la gestion des conflits Je valeurs: "social»vs "économie» Violaine Appel, Université Nancy 2

Internet et réorganisation des pouvoirs Jans les entreprises

F. Assié, J-P. Chaze, L. Schneller, X. Touron. Université Montpellier 1

De l'approche conventionnelle à l'approche communicationnelle des mondes organisationnels

Jean-Luc Bouillon. Université Toulouse 3

Construction et modes de stabilisation d'un nouveau monde organisationnel: la relation actionnaire l'entreprise communicant Sylvie Bourdin. Université Toulouse I. Catherine Loneux, Université Rennes 2

Modes de communication des cadres de santé et l'hôpital. Entre impératifs de normalisation du travail et exigences $d u$ face à face Paule Bourret, Université Montpellier 3

La structuration mutuelle des modes de collaboration et des systèmes d'information dans les organisations californiennes (San Francisco et Bay Area)

Mikaël Gleonnec. EDF Recherche et Développement

La communication de recrutement des effectifs étudiants à l'Université Laval : quelques a priori au recours à des relations de liens dans un projet institutionnel

Alain Lavigne, Université Laval, Québec (Canada)

Nouvelles recherches sur le modèle de « Mohe » dans les entreprises sino-françaises

Zhijié Wang, Université de Wuhan et IUT d'Evry

14.00 Axe 3 : le registre du projet, qui construit des solidarités opérationnelles et fonctionnelles 
Conférence introductive par Rolande Marciniak, professeur de Gestion à l'Université des Sciences économiques et de Gestion de Nantes, secrétaire de rédaction de la revue du Management de Projet, La Cible.

Deux ateliers simultanés dans lesquels seront réparties les communications suivantes :

Lien, sens, action: vers une communication engageante Françoise Bernard. Université de Provence

\section{Technologies de l'information et travail collectif médiatisé. Repenser la relation interpersonnelle.}

Isabelle Comtet. Université Grenoble 3

Le projet comme outil de communication : le cas d'une agence de publicité au Luxembourg

Louise Courtemanche, Cabinet Matrix Consulting. Luxembourg (Grand Duché de Luxembourg)

Le Gemba Kaisen, un moyen d'exister dans l'entreprise Marie-Agnès de Gail. Université de Bretagne Sud

La réduction de l'incertitude dans les organisations industrielles Anne-Hélène Le CornecUbertini. Université de Bretagne Sud

Organisations contemporaines et communication vis et sur les TIC. Le cas des projets d'implantation de progiciels de gestion intégrée Anne Mayère, Université Toulouse 3

La coordination dans les structures projets. Quelles ressources pour quelles coordinations?

Vincent-Bernard Nicotri. IUT Arles

«Morcellisation » d'organisation, unités autonomes, efficacité et permanence de l'action : l'exemple de l'UAG

Bruno Ollivier, Olivier Pulvar. Université des Antilles-Guyane

\subsection{Conclusions du colloque}

Ce programme appelle quelques commentaires. D'abord, nous avons voulu donner aux participants le temps nécessaire à la réflexion. En accordant des plages longues à chaque thème, en laissant des temps de pause, donc de rencontre informelle, significatifs. Ensuite, la dimension internationale est évidente. Non seulement en raison de la forte délégation de l'Institut du Journalisme et de la Communication de l'Université de Wuhan avec lequel nous entretenons un véritable partenariat depuis presque une décennie, mais encore par la présence d'intervenants du Québec, de Belgique, du Brésil et du Grand Duché du Luxembourg. Notre ultime remarque permettra de souligner une autre mixité, celle qui réunit des chercheurs de statut universitaire et d'autres venant de l'entreprise, qu'il s'agisse de consultants ou de professionnels de Recherche et Développement. De ce point de vue. la présence à nos côtés de la revue L'Expression d'entreprise est à la fois précieuse et symbolique. Précieuse parce qu'elle rappelle des souvenirs déjà anciens mais toujours agréables qui évoquent la participation de Patrice Legendre à des colloques que nous avons organisés naguère. Directeur général à la fois de ce fleuron de la presse technique et professionnelle et du Top Com qui rassemble les directeurs de communication des plus grandes structures françaises. Patrice Legendre a été l'un des premiers à considérer qu'il ne devait pas y avoir, qu'il ne pouvait pas y avoir, de coupure entre la recherche universitaire et l'exercice professionnel de la communication. Nous le pensons aussi, nous qui collaborons depuis une dizaine d'années avec une association de chargés de communication, "Communication et Réflexion », qui réunit une trentaine de praticiens, bordelais et lyonnais, soucieux de réfléchir à leur pratique. Symbolique aussi 
en ce sens qu'elle témoigne précisément de cette indispensable ouverture de l'université sur le monde économique.

En plein accord avec la vingtaine de chercheurs qui composent le GREC/O, j'ai déjà exprimé ce qui fait sens pour nous dans un texte que j'ai intitulé « Une certaine idée de la recherche $»^{1}$. Disons simplement que nous pensons que la recherche en sciences humaines et sociales est doublement désintéressée. D'abord parce qu'elle doit s'entreprendre et se mener en dehors de toute idéologie. Ensuite parce qu'elle doit mettre ses résultats à la disposition de la société pour que celle-ci en tire le meilleur parti dans sa marche vers le progrès social. Nous avons beaucoup de chance de pouvoir trouver dans la pratique de la recherche une possibilité d'épanouissement personnel. La moindre des choses est que nous contribuions, modestement sans doute mais réellement, à l'épanouissement des autres.

\section{NOTES}

1. Voir le site Internet du GREC/O : http://www.montaigne.u-bordeaux.fr/GRECO/

\section{AUTEUR}

HUGUES HOTIER

Directeur du GREC/O 